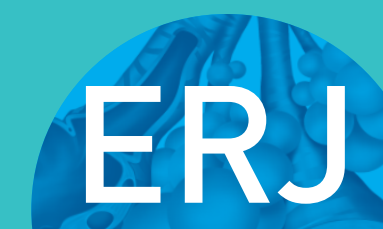

open research
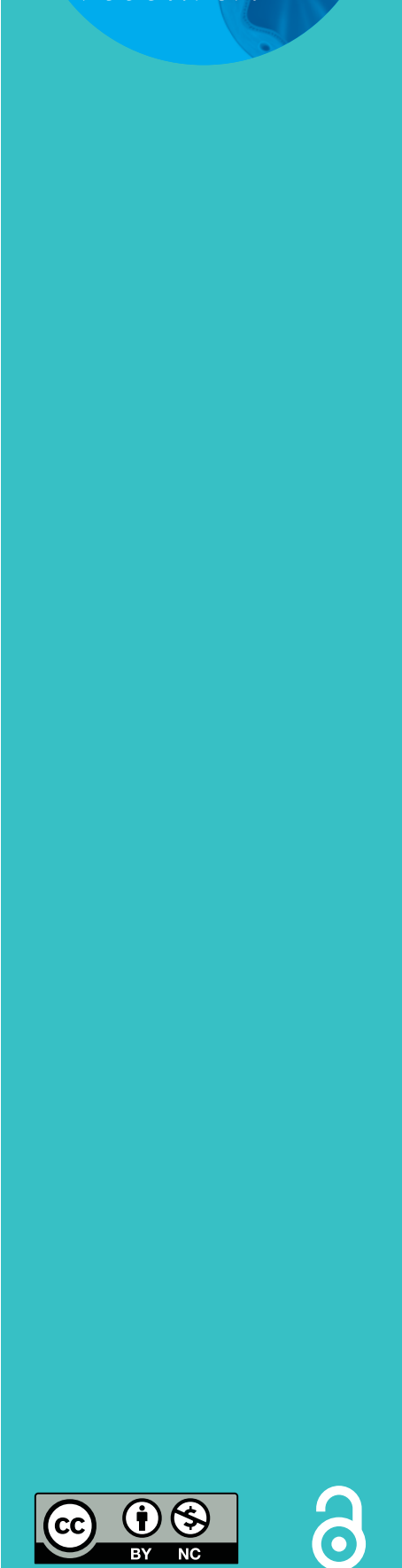

\section{Transbronchial cryobiopsy in interstitial lung disease: experience in 106 cases - how to do it}

\author{
Antonio Bango-Álvarez ${ }^{1}$, Miguel Ariza-Prota (1) ${ }^{1}$, Hector Torres-Rivas², \\ Luis Fernández-Fernández ${ }^{2}$, Amador Prieto $^{3}$, Inmaculada Sánchez ${ }^{4}$, Maria Gil ${ }^{4}$ \\ and Ana Pando-Sandoval ${ }^{5}$
}

\begin{abstract}
Affiliations: ${ }^{1}$ Division of Respiratory Medicine, Hospital Universitario Central de Asturias, Oviedo, Spain. ${ }^{2}$ Division of Pathology, Hospital Universitario Central de Asturias, Oviedo, Spain. ${ }^{3}$ Division of Radiology, Hospital Universitario Central de Asturias, Oviedo, Spain. ${ }^{4}$ Division of Nursery, Hospital Universitario Central de Asturias, Oviedo, Spain. ${ }^{5}$ Division of Respiratory Medicine, Hospital Valle del Nalón, Langreo, Asturias, Spain.
\end{abstract}

Correspondence: Miguel Ariza Prota, Division of Respiratory Medicine, Hospital Universitario Central de Asturias, Avenida de Roma, S/N, 33011 Oviedo, Asturias, Spain. E-mail: arizamiguelahotmail.com

ABSTRACT Transbronchial biopsy using forceps (TBB) is the first diagnostic technique performed on patients with interstitial lung disease (ILD). However, the small size of the samples and the presence of artefacts in the tissue obtained make the yield variable.

Our objectives were 1) to attempt to reproduce transbronchial cryobiopsy under the same conditions with which we performed conventional TBB, that is, in the bronchoscopy unit without intubating the patient and without fluoroscopy or general anaesthesia; 2) to describe the method used for its execution; and 3) to analyse the diagnostic yield and its complications.

We carried out a prospective study that included 106 patients with clinical and radiological features suggestive of ILD who underwent cryo-transbronchial lung biopsy (cryo-TBB) under moderate sedation without endotracheal intubation, general anaesthesia or use of fluoroscopy. We performed the procedure using two flexible bronchoscopes connected to two video processors, which we alternated until obtaining the number of desired samples.

A definitive diagnosis was obtained in 91 patients (86\%). As for complications, there were five pneumothoraces $(4.7 \%)$ and in no case was there severe haemorrhage or exacerbation of the underlying interstitial disease. Cryo-TBB following our method is a minimally invasive, rapid, safe and economic technique that can be performed in a bronchoscopy suite under moderate sedation without the need for intubating the patient or using fluoroscopy and without requiring general anaesthesia.

$@$ ERSpublications

Transbronchial cryobiopsy in interstitial lung disease http://ow.ly/jz1F309tMyf

Cite this article as: Bango-Álvarez A, Ariza-Prota $\mathrm{M}$, Torres-Rivas $\mathrm{H}$, et al. Transbronchial cryobiopsy in interstitial lung disease: experience in 106 cases - how to do it. ERJ Open Res 2017; 3: 00148-2016 [https://doi.org/10.1183/23120541.00148-2016].

Received: Dec 092016 | Accepted after revision: Feb 092017

Conflict of interest: None declared.

Copyright $\odot$ ERS 2017. This article is open access and distributed under the terms of the Creative Commons Attribution Non-Commercial Licence 4.0. 


\section{Introduction}

The diagnosis of diffuse interstitial lung disease is performed by a multidisciplinary team that involves pulmonologists, radiologists and pathologists. Close collaboration between these different areas of expertise is required to define which patients will need a biopsy and those in whom biopsy is not necessary [1].

Surgical pulmonary biopsy continues to be considered the gold standard for recognising histological patterns and aiding the pulmonologist in considering possible causes of disease [2,3]. Conventional transbronchial biopsy using forceps (TBB) serves to diagnose sarcoidosis, neoplasias, some infections and cryptogenic organising pneumonia (COP) [1]. However, the small size of the samples obtained and the artefacts produced by the forceps on the tissue makes this insufficient in a large number of patients [1-4]. Recently, studies [5] have been published that confirm the efficacy of new treatments, such as pirfenidone, in patients with idiopathic pulmonary fibrosis (IPF). The early diagnosis of disease is thus important.

With transbronchial cryobiopsies carried out using cryoprobes, the quality of samples obtained and the diagnostic yield are improved compared with transbronchial biopsies with forceps [6, 7]. However, the requirement to perform it in an operating room with general anaesthesia and tracheal intubation [8-10] makes it closer to being a surgical technique than conventional TBB performed by a pulmonologist.

\section{Patients and methods}

A prospective study was conducted in the Central University Hospital of Asturias (Hospital Universitario Central de Asturias - HUCA) from June 2013 to December 2015 and was approved by the ethics committee of the hospital. Patients were referred to the Unit of Interventional Bronchoscopy and Pleura of the hospital after being assessed by the Multidisciplinary Committee on Diffuse Interstitial Lung Diseases, which is composed of pulmonologists, radiologists, pathologists and rheumatologists who are experts on autoimmune diseases. We enrolled patients who had clinical and radiographic features of interstitial lung disease (ILD) in high-resolution computed tomography (HRCT), which was inconsistent with usual interstitial pneumonitis (UIP). Patients with images typical of UIP in HRCT according to the British Thoracic Society guidelines were excluded. Cryo-transbronchial lung biopsies (cryo-TBBs) were performed in the most affected lobes according to imaging studies of the chest, following the recommendations of the guidelines [1-4], although always with a preference for the lower lobes for greater safety. Two pathologists assessed the tissue samples and agreed on the histopathologic diagnoses. The tissue quality variables recorded were tissue sample size (diameter and area), the presence of pleural tissue, and the percentage of artefact-free tissue in each sample. Signs of atelectasis, tissue sample fragmentation and clots were considered artefacts; the pathologists noted these and then recorded how much of each sample was free of such artefacts. The duration times of reintubation of patients' airways with the second bronchoscope and the complete time for the procedure were recorded. Bleeding was collected into a mucus resorvoir (Mocstrap; Proclinics, Barcelona, Spain), which has a scale in millilitres, and quantified according to the following classification: mild haemorrhage, bleeding $<10 \mathrm{~mL}$; moderate, $10-40 \mathrm{~mL}$; severe, $>40 \mathrm{~mL}$ (table 1). In all patients, the protocol included complete lung

\section{TABLE 1 Procedural details of patients who underwent cryo-transbronchial lung biopsy}

\begin{tabular}{|c|c|}
\hline \multicolumn{2}{|l|}{ Localisation } \\
\hline RLL & $46(43 \%)$ \\
\hline $\mathrm{RLL}$ and $\mathrm{ML}$ & $17(16 \%)$ \\
\hline LLL and LUL & $20(18 \%)$ \\
\hline LLL & $21(20 \%)$ \\
\hline LUL & $2(1 \%)$ \\
\hline \multicolumn{2}{|l|}{ Bleeding } \\
\hline Mild $<10 \mathrm{~mL}$ & $89(84 \%)$ \\
\hline Moderate $10-40 \mathrm{~mL}$ & $17(16 \%)$ \\
\hline Severe $>40 \mathrm{~mL}$ & $0(0 \%)$ \\
\hline Length of procedure min & $20(12-25)$ \\
\hline Length of reintubation with second bronchoscope $s$ & $8(5-15)$ \\
\hline Number of samples & $3(1-5)$ \\
\hline Diameter $\mathrm{mm}$ & $5.1 \pm 1.8$ \\
\hline Area $\mathrm{mm}^{2}$ & $15.7 \pm 9$ \\
\hline Presence of pleural tissue & 0 \\
\hline $\mathbf{7 5} \%$ artefact-free $\%$ of total sampled area & $75.2 \%$ \\
\hline Pneumothorax & $5(4.7 \%)^{\#}$ \\
\hline
\end{tabular}


function tests, HRCT, complete blood count, coagulation and echocardiogram. We excluded patients who presented with blood dyscrasias, severe respiratory failure or unstable heart diseases, anticoagulant medication, pulmonary hypertension, forced expiratory volume in $1 \mathrm{~s}$ (FEV1) of $<40 \%$, forced vital capacity (FVC) of $<50 \%$ and diffusing capacity of the lung for carbon monoxide (DLCO) of $<40 \%$. All patients and the pulmonologist who performed the procedure signed an informed consent form.

\section{Bronchoscopy procedure and cryo-TBB procedure}

All procedures were performed in the bronchoscopy suite with supplemental oxygen delivery through a nasal catheter placed in the larynx above the vocal cords, or sometimes intratracheally (figure 1a). The patient was placed in the anti-Trendelenburg position, and continuous records of oxyhaemoglobin saturation, blood pressure, heart rate, respiratory rate and ECG were made. Local anaesthesia of the upper and lower airway was performed by instilling lidocaine through a catheter (Perifix-Braun Medical, Bethlehem, PA, USA) introduced in the working channel of the bronchoscope (figure $1 \mathrm{~b}$ ). Sedation was performed with midazolam $\left(0.07 \mathrm{mg} \cdot \mathrm{kg}^{-1}\right)$ and fentanyl citrate $\left(0.5-2 \mu \mathrm{g} \cdot \mathrm{kg}^{-1}\right)$, starting with boluses of $1-3 \mathrm{mg}$ of midazolam and $0.1 \mu \mathrm{g}$ of fentanyl citrate. Sedation was maintained with intermittent boluses of $1.2 \mathrm{mg}$ midazolam and $0.1 \mu \mathrm{g}$ fentanyl citrate according to the clinical judgment of the pulmonologist who directed the team of the unit, which was composed of two interventional pulmonologists, two expert nurses and one nursing assistant. Two flexible bronchoscopes connected to two different video processors were used (figure 1c).

The first bronchoscope (model EB 1975K, with a $2.8 \mathrm{~mm}$ working channel diameter), connected to one of the two processors, was introduced through a short bite block (figure 1d) until the desired segment was located correctly to perform biopsies. The flexible cryoprobe $(1.9 \mathrm{~mm}$ diameter and $900 \mathrm{~mm}$ length; figure 2a), connected to cryotherapy equipment (Erbokryo ${ }^{\oplus} \mathrm{Ca}$, Erbe, Germany; figure 2b), was introduced into the working channel of the bronchoscope, moving forward distally until it was noted that the cryoprobe could not advance further. The cryoprobe was gently retracted by approximately $1-2 \mathrm{~cm}$, as determined by the marks on the distal area of the probe (figure 2c). With the probe in that position, the Erbokryo pedal was pressed for $5 \mathrm{~s}$, freezing the probe point and causing the tissue sample to adhere to the probe. This sample was extracted along with the bronchoscope for thawing and subsequent processing (figure $2 \mathrm{~d}$ ). The second bronchoscope (model EB $1970 \mathrm{TK}$ with a $3.2 \mathrm{~mm}$ working channel) was immediately introduced, and was connected to the second processor and prepared for reintroduction into the patient's airways in a manoeuvre that lasted $5-10 \mathrm{~s}$ and allowed immediate control of any haemorrhage resulting from the cryobiopsy by suction or by instilling substances such as adrenaline, ice-cold saline or amchafibrin. If there were no complications with this second bronchoscope, then we obtained the second sample. The two bronchoscopes were alternated until the desired number of samples (two to five) was obtained.
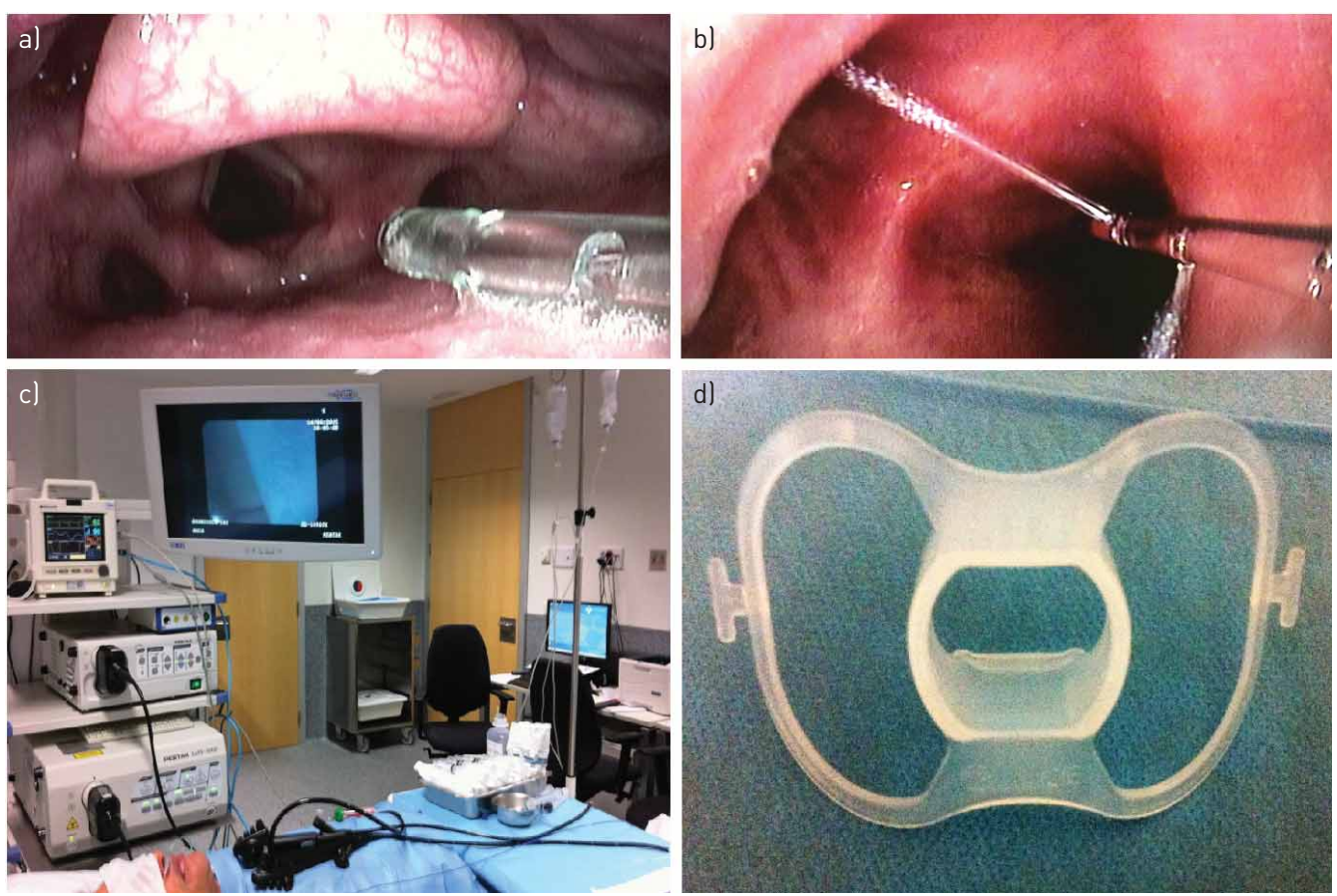

FIGURE 1 a) Nasal catheter placed in the larynx above the vocal cords or sometimes intratracheally. b) Instillation of lidocaine through a Perifix catheter introduced in the working channel of the bronchoscopy.

c) Our two flexible bronchoscopes connected to two different video processors. d) Short oral biter. 

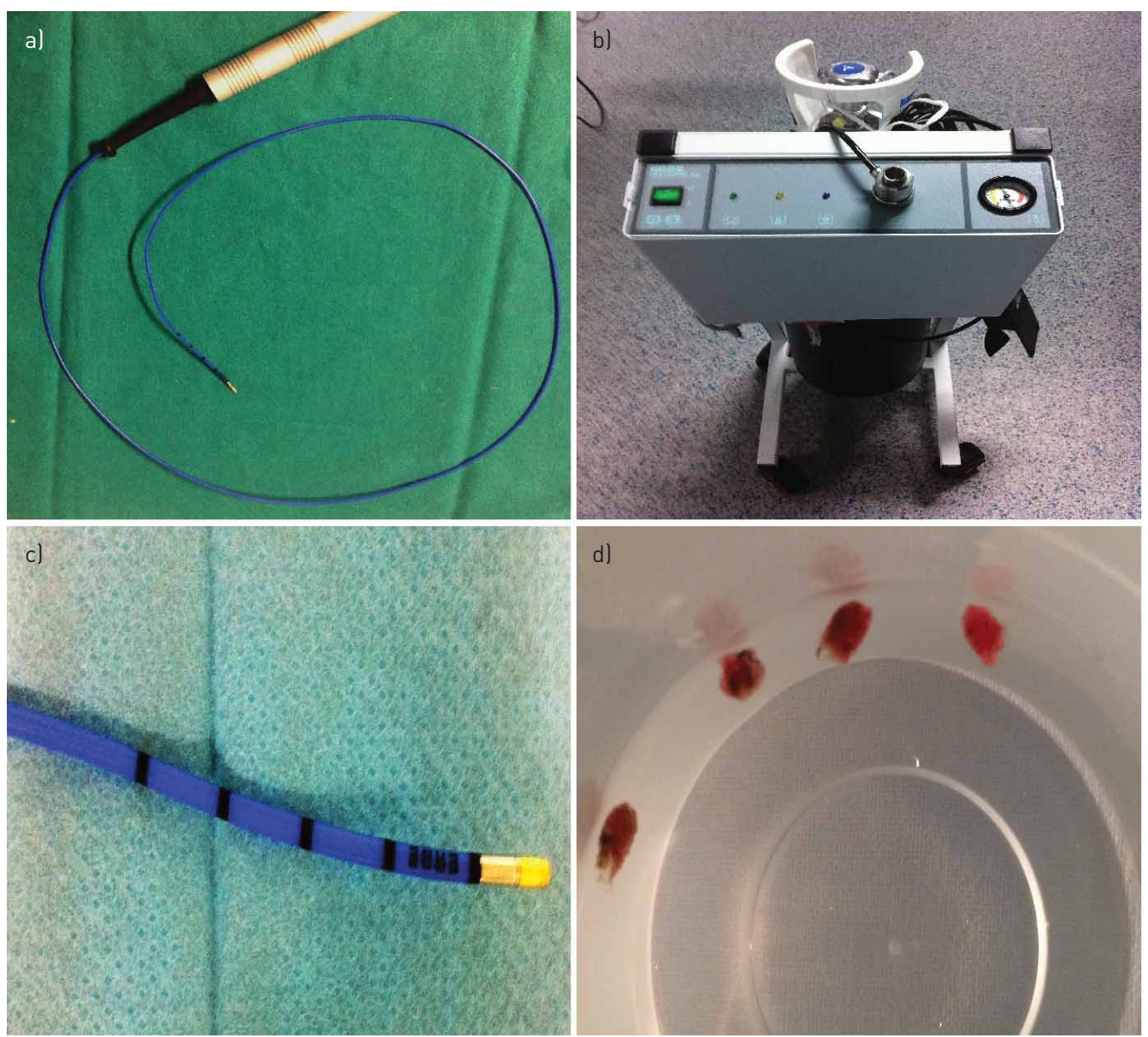

FIGURE 2 a) The flexible $1.9 \mathrm{~mm}$ diameter and $900 \mathrm{~mm}$ length cryoprobe, b) connected to the cryotherapy equipment. c) Marks on the distal area of the probe $(1 \mathrm{~cm}$ between each mark). d) Cryo-transbronchial lung biopsy samples from one of the patients.

When it was confirmed that no bleeding had occurred and that the patient was well controlled, the procedure was stopped. Chest radiography or pleural echography was then performed to confirm that a pneumothorax had not taken place, and the patient was discharged $2 \mathrm{~h}$ after verifying that there were no complications. Follow-up was conducted on all patients at $24 \mathrm{~h}$ via phone call to check that there were no delayed complications.

\section{Results}

During the study period, 106 patients with ILD in HRCT were included for cryo-TBBs. In all patients, a bronchoalveolar lavage was performed before cryobiopsies in the most affected pulmonary lobe. Table 2 shows the general characteristics of the patients, while table 1 presents the procedure characteristics. In 37 patients (35\%), cryo-TBBs were performed in more than one lobe, and in all patients cryo-TBBs were performed in at least two segments of the same pulmonary lobe.

There were no severe complications in our series of patients. A mild haemorrhage $(<10 \mathrm{~mL})$ occurred in $84 \%$ of patients, and $16 \%$ had moderate haemorrhage $(10-40 \mathrm{~mL})$. No patient had a severe haemorrhage that required stopping the procedure and defining special measures, such as admission to the intensive care unit, surgical treatment, transfusions and so on. We had five patients with pneumothorax (4.7\%), of which three were resolved with conservative measures without requiring admission and another two required a pleural drainage tube and admission for $48 \mathrm{~h}$.

We obtained an average of three biopsies per patient, with an average diameter of $5.1 \mathrm{~mm}$ (figure $2 \mathrm{~d}$ ) and a mean specimen area of $15.7 \pm 9 \mathrm{~mm}^{2}$, always trying to obtain a representative sample from each segment of the targeted lobe. The average time per procedure was $9.7 \mathrm{~min}$, and the average reintubation time with the second bronchoscope was $8.5 \mathrm{~s}$. In all patients, samples were obtained with a representation of pulmonary parenchyma. A definitive diagnosis after consensus of the multidisciplinary committee was obtained in 91 patients (86\%) (table 3), and there was no change in the histopathological diagnoses 


\section{TABLE 2 Patient characteristics at baseline}

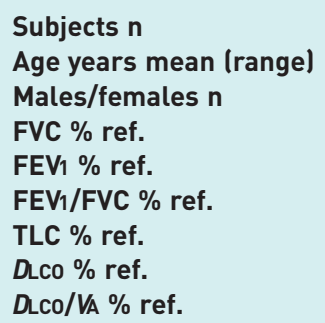

Data are presented as mean \pm SD unless otherwise stated. FVC: forced vital capacity; $\%$ ref.: $\%$ of reference value; FEV1: forced expiratory volume in $1 \mathrm{~s}$; TLC: total lung capacity; DLCo: carbon monoxide diffusing capacity of the lung; VA: alveolar volume.

informed by the pathologists after being assessed by the multidisciplinary committee. In three $(2.8 \%)$ of the 15 patients in whom transbronchial cryobiopsies were inconclusive, a surgical biopsy (video-assisted thoracoscopic surgery (VATS)) was performed, with the final diagnoses being mild interstitial fibrosis, unclassifiable interstitial pneumonia, and desquamative interstitial pneumonitis (the patient had a prolonged air leak as a complication of surgery). In the 12 remaining patients, cryobiopsy served to rule out other processes such as neoplasia, infection, granulomatosis or drug toxicity, and it was decided to conduct conservative follow-up as surgical biopsy was contraindicated by comorbidities and the increased risk they presented. The most common diagnoses of our series were as follows: idiopathic pulmonary fibrosis (IPF/UIP) $(n=22)$, cryptogenic obstructive pneumonia (COP) $(n=10)$, nonspecific interstitial pneumonia (NSIP) $(n=11)$, and silicosis $(n=12)$. The pathological anatomy of a desquamative pneumonitis (figure $3 \mathrm{a}-\mathrm{c}$ ) and a pneumoconiosis (figure $3 \mathrm{~d}-\mathrm{f}$ ), common in our geographic area, are shown.

\section{Discussion}

This study describes a new procedure for performing cryo-TBBs in ILD. We have demonstrated that the cryo-TBB used for the diagnosis of ILD can be performed in an adequately prepared bronchoscopy suite equipped with the necessary means [11] without needing to intubate the patient, without fluoroscopy and with moderate sedation. This procedure consists of using two flexible bronchoscopes connected to two video processors that we alternated by the oral route until obtaining the desired number of samples.

The alternation of two bronchoscopes by the oral route permits the procedure to be rapid and allows for any haemorrhages produced by cryobiopsy to be controlled immediately, because we are able to reintubate the patient within a few seconds. When this is conducted by a single bronchoscope through the nose, the time that it remains outside the airway is greater than $1 \mathrm{~min}$, which is how long the cyroprobe takes to

\section{TABLE 3 Final diagnosis after multidisciplinary review}

Hypersensitivity pneumonitis

Eosinophilic pneumonia

Idiopathic pulmonary fibrosis

Diffuse alveolar damage

Silicosis

Desquamative interstitial pneumonia

Cryptogenic organising pneumonia

Drug-induced interstitial lung disease

Respiratory-bronchiolitis interstitial lung disease

Sarcoidosis

Nonspecific interstitial pneumonia

Adenocarcinoma

Lymphocytic interstitial pneumonia

Normal lung tissue

Inconclusive

Total

Data are presented as number of subjects. 

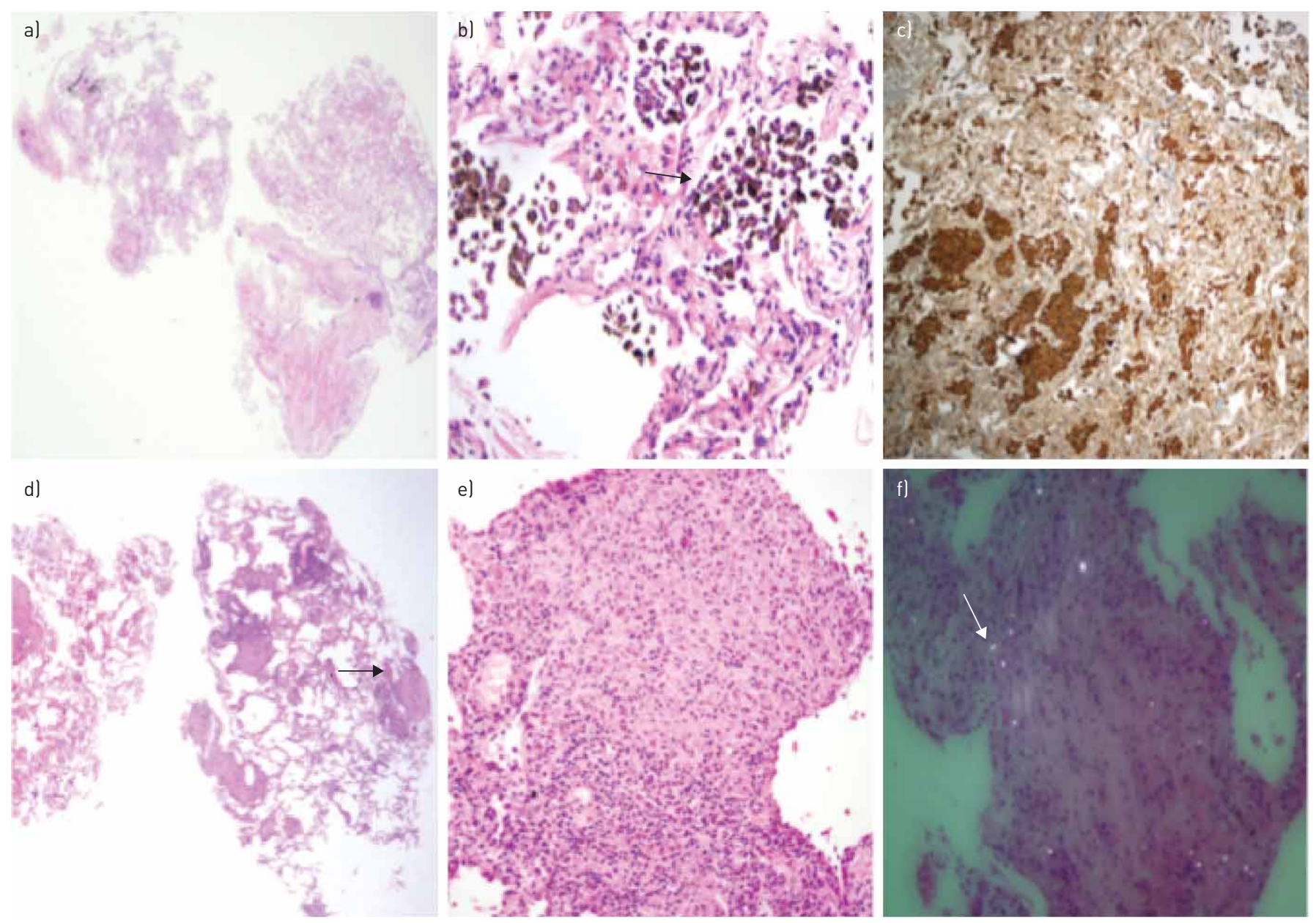

FIGURE 3 A 45-year-old smoking man diagnosed with desquamative interstitial pneumonia (DIP) by a) cryo-transbronchial lung biopsy (haematoxylin-eosin stain) showing b) thick alveolar septa filled by numerous macrophages with c) CD68 staining. A 67-year-old man diagnosed with silicosis by d) cryo-transbronchial lung biopsy showing e) a silicotic nodule (black arrow) and f) silica crystals with polarised light (white arrow).

thaw and release the cryobiopsy adhered to it. If a haemorrhage were produced, we would not be able to control it immediately. In addition, the cryobiopsy could be released upon removing the bronchoscope along the narrow nasal passage and thus lost [12].

Although our method allows for rapid control of the patient's airway, our greatest concern was always the possible occurrence of complications, especially haemorrhage. To prevent haemorrhage, we selected patients by rigorously excluding those who had a greater risk of having haemorrhages (blood dyscrasias, anticoagulant medication, pulmonary hypertension, among others) and we attempted to perform cryobiopsies in the lower lobes if pulmonary involvement on the HRCT was diffuse, due to the greater time a bronchoscopist has to control a possible haemorrhage in this location than in the upper lobes. As our learning curve progressed, we also included the upper lobes when the more affected lobe in HRCT was located there, always placing the patient in the anti-Trendelenburg position during the entire procedure.

Using this method, haemorrhage in our series was mild or moderate in all patients, and we controlled bleeding with suction, adrenaline, ice-cold saline and amchafibrin. In no patient was there severe haemorrhage (greater than $40 \mathrm{~mL}$ ). We thus believe that the appropriate selection of patients, the experience of the interventional pulmonologist who performed the procedure and of the team that formed the unit, and the haemostasis effect produced by freezing the probe on the tissue are key to our success. It is also important to emphasise that the number of pneumothoraces in our study is lower than in other published studies performed in an operating room under general anaesthesia $[9,10]$ and the yield was better $[8,9]$.

Our study differs from other studies [6-10] in that we did not use a fluoroscopic guide to place the cryoprobe in the small-calibre airway, making our suite radiation-free. Our results regarding cost-effectiveness, complications and lack of pleura in any of the biopsies demonstrate that fluoroscopy is not essential and is most probably unnecessary for this technique. 
Most of the studies published on transbronchial [6-10] or endobronchial [13] cryobiopsies have been performed on intubated patients because the tissue adhered to the cryoprobe cannot be extracted through the working channel of the video bronchoscope and because the bronchoscope and cryoprobe need to be removed as a unit. Under these conditions, it is important to achieve rapid reinsertion of the bronchoscope to control any possible bleeding produced by the cryoprobe, by suction, tamponade, instillation of substances such as adrenaline, ice-cold saline or even an occlusion balloon. Our method allows us to perform all of these manoeuvres in a few seconds, so that the patient's safety is ensured.

The procedure we describe has also allowed us to perform cryobiopsies in endobronchial lesions, with better yield than conventional biopsy with forceps and the same safety, but with the advantage of not having to perform it in an operating room with the patient intubated and under general anaesthesia, which would considerably increase the economic cost. Another advantage of this procedure is that, by not needing general anaesthesia or an operating room, and by performing the procedure in the bronchoscopy suite like other procedures we perform in routine clinical practice, after a training period this technique can be performed by pulmonologists who work in hospitals of different levels of care, greatly amplifying the field of future users of the technique.

There have been in vitro studies in animals demonstrating that the sample size depends on variables such as tissue type, probe diameter, application time and pressure exerted by the probe on the tissue. These studies have shown that even the smallest probes currently used provide larger biopsies than those obtained with a conventional biopsy forcep [14], as used in most bronchoscopy suites at present. FranKE et al. [15], very recently, have published a study in animals that uses a mini probe to obtain samples through the working channel of a bronchoscope without needing to remove the bronchoscope from the central airway. In our experience, the $1.9 \mathrm{~mm}$ diameter probe has provided us with good yield and very few complications. It has a long half-life and it is the probe we advise using at this time, although it would be interesting to conduct comparative studies of the different probes that exist on the market.

To date, the debate on how to best perform the procedure is focused on two factors, the type of sedation and control of the airway; it can be performed with the patient intubated with an endotracheal tube or a rigid tracheoscope under deep sedation or without intubating the patient with conscious sedation [16] and in the bronchoscopy suite. A limitation of our study could be that the patients are in an initial phase of their disease, as can be observed by the lung function values shown in table 2. It is possible that, in more advanced phases of disease (e.g. $\left.\mathrm{FEV}_{1}<40 \%\right)$, the yield and complications of the technique would be different, and we therefore believe that new prospective studies are necessary to standardise all aspects of the procedure [17].

\section{Conclusions}

We conclude that, in well-selected patients with good lung function and without associated risk factors, our procedure is the first choice due to its many advantages and few drawbacks. Cryo-TBB following our method is a minimally invasive, rapid, safe and economic technique that can be performed in a bronchoscopy suite under moderate sedation without the need for intubating the patient or using fluoroscopy, and without requiring general anaesthesia.

\section{Acknowledgements}

M. Ariza-Prota, A. Bango-Álvarez, H. Torres-Rivas, L. Fernández-Fernández, I. Sánchez, M. Gil and A. Prieto performed the research and collected the data. M. Ariza-Prota and A. Bango-Álvarez wrote the manuscript. A. Pando-Sandoval designed the case report design and reviewed the manuscript. M. Ariza-Prota is the guarantor of the paper, taking responsibility for the integrity of the work as a whole, from inception to published article.

\section{References}

1 American Thoracic Society, European Respiratory Society. American Thoracic Society/European Respiratory Society International Multidisciplinary Consensus Classification of the Idiopathic Interstitial Pneumonias. Am J Respir Crit Care Med 2002; 165: 277-304.

2 Travis WD, Costabel U, Hansell DM, et al. An official American Thoracic Society/European Respiratory Society statement: update of the international multidisciplinary classification of the idiopathic interstitial pneumonias. Am J Respir Crit Care Med 2013; 188: 733-748.

3 Raghu G, Collard HR, Egan JJ, et al. An official ATS/ERS/JRS/ALAT statement: idiopathic pulmonary fibrosis: evidence-based guidelines for diagnosis and management. Am J Respir Crit Care Med 2011; 183: 788-824.

4 Xaubet A, Ancochea J, Bollo E, et al. Guidelines for the diagnosis and treatment of idiopathic pulmonary fibrosis. Sociedad Española de Neumología y Cirugía Torácica (SEPAR) Research Group on Diffuse Pulmonary Diseases. Arch Bronconeumol 2013; 49: 343-353.

5 King TE, Bradford WZ, Castro-Bernardini S, et al. A phase 3 trial of pirfenidone in patients with idiopathic pulmonary fibrosis. N Engl J Med 2014; 370: 2083-2092.

6 Pajares V, Puzo C, Castillo D, et al. Diagnostic yield of transbronchial cryobiopsy in interstitial lung disease: a randomized trial. Respirology 2014; 19: 900-906. 
7 Babiak A, Hetzel J, Krishna G, et al. Transbronchial cryobiopsy: a new tool for lung biopsies. Respiration 2009; 78: 203-208.

8 Pajares V, Torrego A, Puzo C, et al. Transbronchial lung biopsy using cryoprobes. Arch Bronconeumol 2010; 46: 111-115.

9 Hernández-González F, Lucena CM, Ramírez J, et al. Utilidad de la criobiopsia en el diagnóstico de la enfermedad pulmonar intersticial difusa: análisis de rentabilidad y coste [Cryobiopsy in the diagnosis of diffuse interstitial lung disease: yield and cost-effectiveness analysis]. Arch Bronconeumol 2015; 51: 261-267.

10 Casoni GL, Tomassetti S, Cavazza A, et al. Transbronchial lung cryobiopsy in the diagnosis of fibrotic interstitial lung diseases. PLoS ONE 2014; 9: e86716.

11 Flandes J, Alfageme I. Recursos humanos, físicos, de material y terapeúticos [Human, physical, material, and therapeutic resources]. In: Necesidades y Organización de Una Unidad de Endoscopia Respiratoria [Necessities and Organization of a Respiratory Endoscopy Unit]. Madrid, Luzán Ediciones, 2002; pp. 9-19.

12 Fruchter O, Fridel L, El Raouf BA, et al. Histological diagnosis of interstitial lung diseases by cryo-transbronchial biopsy. Respirology 2014; 19: 683-688.

13 Hetzel J, Eberhardt R, Herth FJ, et al. Cryobiopsy increases the diagnostic yield of endobronchial biopsy: a multicentre trial. Eur Respir J 2012; 39: 685-690.

14 Franke KJ, Szyrach M, Nilius G, et al. Experimental study on biopsy sampling using new flexible cryoprobes: influence of activation time, probe size, tissue consistency, and contact pressure of the probe on the size of the biopsy specimen. Lung 2009; 187: 253-259.

15 Franke KJ, Linzenbold W, Nuessle D, et al. A new tool for transbronchial cryobiopsies in the lung: an experimental feasibility ex vivo study. Respiration 2016; 91: 228-234.

16 Gasparini S, Bonifazi M. Cryobiopsy for interstitial lung diseases. J Bronchol Interv Pulmonol 2016; 23: 4-6.

17 Poletti V, Hetzel J. Transbronchial cryobiopsy in diffuse parenchymal lung disease: need for procedural standardization. Respiration 2015; 90: 275-278. 